\title{
EEG De-noising using SURE Thresholding based on Wavelet Transforms
}

\author{
G. Geetha \\ Research Scholar \\ Department of Computer Science \\ Avinashilingam Deemed University for Women \\ Coimbatore, India
}

\author{
Dr.S.N.Geethalakshmi \\ Associate Professor \\ Department of Computer Science \\ Avinashilingam Deemed University for Women \\ Coimbatore, India
}

\begin{abstract}
The Electroencephalogram (EEG) is a biological signal that represents the electrical activity of the brain. However, the presence of artifacts like electro-oculogram (EOG), Electrocardiogram (ECG), electromyogram (EMG) and powerline noise in the EEG signal is a major problem in the study of brain potentials. Hence, these superfluous signals are needed to be removed. There are various methods for removal of artifacts. This paper discusses a wavelet-based approach for correcting the artifacts generated by eye blinks, eyeball movements and facial muscle movements in EEG.
\end{abstract}

\section{Keywords}

Wavelets, Ocular Artifacts (OA), Muscular Artifacts (MA)

\section{INTRODUCTION}

Electroencephalogram (EEG) is a bioelectric brain activity used as an important tool by physicians for studying the functional state of the brain and for diagnosing certain neurophysiologic states and disorders. It is also used as a non-invasive approach for research in the study of neurophysiology. The presence of physiological artifacts such as eye blinks, muscular movement, and cardiac pulses in EEG recordings obscure the underlying processes and makes analysis problematic. Eye movement produces electrical activity the EOG which is strong enough to be clearly visible in the EEG [7,8]. The EOG reflects the potential difference between the cornea and the retina which changes during eye movement. Another common artifact is caused by eyelid movement i.e. eye blinks which also influences the corneal-retinal potential difference. The blinking artifact usually produces a more abruptly changing waveform than eye movement, and, accordingly, the blinking artifact contains more high-frequency components. . Eye-blinks and movement of the eyeballs produce electrical signals that are collectively known as Ocular Artifacts (OA)[3]. Another common artifact caused by electrical activity of contracting muscles, measured on the body surface by the EMG. This type of artifact is primarily encountered when the patient is awake and occurs during swallowing, grimacing, jaw clenching, frowning, chewing, talking, sucking, and hiccupping. These artifacts are often called as Muscular Artifacts (MA). These are of the order of milli-volts and they contaminate the EEG $[9,10]$ signals which are of the order of micro-volts. The frequency range of EEG signal is 0 to $64 \mathrm{~Hz}$ and the OA occur within the range of 0 to $16 \mathrm{~Hz}$ and that of MA occur in the range of 50 to $500 \mathrm{~Hz}$. If to the wavelet based EOG and EMG signals, correction algorithm is applied to the entire length of the EEG signal, it results in the threshold of both low frequency and high frequency components even in the non-OA zones. Due to the overlapping of these artifacts over the desired signals, there is a considerable loss of valuable background EEG activity. Though the detection of OA and MA zones can be done by visual inspection, the OA and MA time zones need to be given as input to the EOG and EMG correction procedure, which is a laborious process. Hence there is a need for automatic detection of artifact zones. This paper discusses a method to automatically identify OA and MA zones and applying wavelet based adaptive threshold algorithm only to the identified OA zones, which avoids the removal of background EEG information. Adaptive thresholding applied only to the OA and MA zone does not affect the low frequency components in the non-OA zones and high frequency components in the nonMA zones ,i.e., it preserves the shape (waveform) of the EEG signal in non-artifact zones which is of very much importance in clinical diagnosis.

\section{WAVELETS FOR ANALYZING EEG SIGNALS}

A 'wavelet' is a small waveform which has its energy concentrated in time. Wavelet Transforms [1] are used to convert a signal into a series of wavelets. The wavelet transform is an important tool for analysis of EEG signals. One of the primary benefits of the wavelet transform is that it is localized in both time and frequency, whereas other classical methods like the Fourier transform are localized in frequency, only. Moreover, the wavelet transform offers good time resolution for low-frequency components and good frequency resolution for high-frequency components of the signal being analyzed. It overcomes shortcomings of other similar methods, such as the short-time Fourier transform, wherein time-frequency localization is constant for all frequencies. The result is that a wavelet transform can be designed to detect specific signal transitions localized in time and frequency. Continuous wavelet transform (CoWT) is powerful in singularity detection. A discrete and fast implementation of CoWT (generally with real valued basis) is known as the standard DWT (Discrete Wavelet Transform). With standard DWT, signal has a same data size in transform domain and therefore it is a non-redundant transform. Standard DWT can be implemented through a simple filter bank structure of recursive FIR filters. A very important property; Multiresolution Analysis (MRA) allows DWT to view and process different signals at various resolution levels. The advantages such as non-redundancy, fast and simple implementation with digital filters using micro-computers, and MRA capability popularized the DWT for signal denoising.

$$
\Psi_{j . k}(t)=2^{j / 2} \Psi\left(2^{j}-k\right)
$$

The Discrete Wavelet Transform (DWT) means, choosing subsets of the scales ' $\mathrm{j}$ ' and positions ' $\mathrm{k}$ ' of the mother wavelet $\psi(\mathrm{t})$.Choosing scales and positions are based on powers of two, 
which are called dyadic scales and positions ( $\mathrm{j}$ and $\mathrm{k}$ are integers ). Equation (1) shows that, it is possible to build a wavelet for any function by dilating a function on $\psi(\mathrm{t})$ with a coefficient $2 \mathrm{j}$, and translating the resulting function on a grid whose interval is proportional to $2-\mathrm{j}$. Contracted (compressed) versions of the wavelet function match the high-frequency components, while dilated (stretched) versions match the lowfrequency components. By correlating the original signal with wavelet functions of different sizes, the details of the signal can be obtained at several scales. These correlations with the different wavelet functions can be arranged in a hierarchical scheme called multi-resolution decomposition. The multiresolution decomposition algorithm [2] separates the signal into "details"at different scales and a coarser representation of the signal named "approximation".

DWT (Discrete Wavelet Transform), being non-redundant, is a very powerful tool for many non-stationary Signal Processing applications, but it suffers from three major limitations; 1) shift sensitivity, 2) poor directionality, and 3) absence of phase information. To reduce these limitations, many researchers developed real-valued extensions to the standard DWT such as SWT (Stationary Wavelet Transform). The key point is that it gives a better approximation than the discrete wavelet transform (DWT) since, it is redundant, linear and shift invariant [2]. These properties provide the SWT to be realized using a recursive algorithm. Therefore, the SWT is very useful algorithm for analyzing a linear system. A brief description of the SWT is presented here.

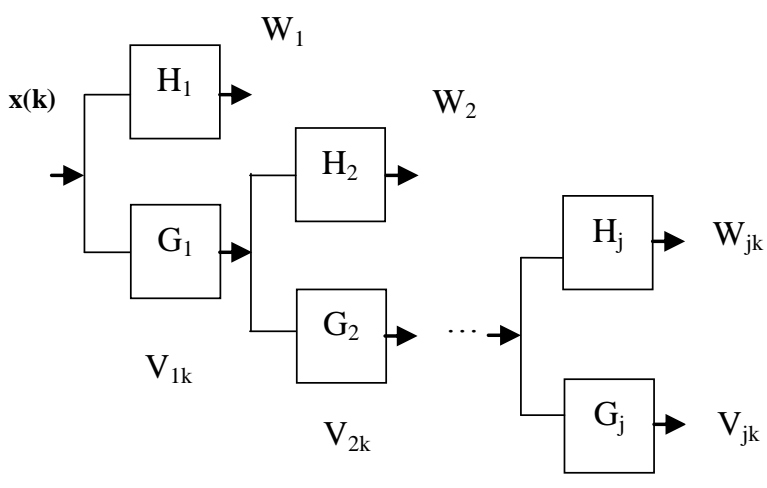

Fig 1

\section{METHODOLOGY}

Figure 1 shows the computation of the SWT of a signal $x(k)$, where $\mathrm{Wj}, \mathrm{k}$ and $\mathrm{Vj}, \mathrm{k}$ are called the detail and the approximation coefficients of the SWT. The filters $\mathrm{Hj}$ and $\mathrm{Gj}$ are the standard low pass and high pass wavelet filters, respectively. In the first step, the filters $\mathrm{H} 1$ and $\mathrm{G} 1$ are obtained by up sampling the filters using the previous step (i.e. $\mathrm{Hj}-1$ and $\mathrm{Gj}-1$ ) [6]. The detail coefficients $\mathrm{Wj}, \mathrm{k}$ are equal to the output of the highpass filters and similarly the approximation coefficients $\mathrm{Vj}, \mathrm{k}$ are equal to the output of the lowpass filters. According to the time frequency properties of the wavelet transform $\mathrm{Hj}$ and $\mathrm{Gj}$ are a bank of ideal narrowband filters.

The EEG recordings are contaminated by EOG and EMG signals. The EOG and EMG signals are non-cortical activities. The eye, facial muscles and brain activities have physiologically separate sources, so the recorded EEG is a superposition of the true EEG and some portion of the EOG signal and EMG signals [1]. It can be represented as

$$
\mathrm{EEG}_{\text {rec }}(\mathrm{t})=\mathrm{EEG}_{\text {true }}(\mathrm{t})+\mathrm{s} \cdot \mathrm{EOG}(\mathrm{K})+\mathrm{t} \cdot \mathrm{EMG}(\mathrm{K})
$$

where,

EEGrec(t )- Recorded EEG which is contaminated signal and holds artifacts

EEGtrue(t)- EEG due to the cortical activity (i.e., Brain activity)

s.EOG(k )- Propagated ocular artifact due to eye blinks and movements, and having impact over the recording site

t.EMG(K)- Propagated muscular artifact due to eye blinks, jaw clenching, swallowing spit which again reflects over the recording site

EEGtrue(t )-is to be estimated from by efficiently removing unwanted artifacts s.EOG(k) and t.EMG(K) and at the same time retaining the EEG activity

The Algorithm proposed in this paper involves the following steps:

i. Apply stationary wavelet transform to the contaminated EEG signals and decompose it up to six levels with Coiflet (Coif5) as a basis function.

ii. Identify the spikes in the contaminated EEG at each level.

iii. Identify the ocular and muscular artifact zones using coefficient of variation.

iv. Apply de-noising technique (equation 4). To fix the suitable threshold value and threshold function for the artifact zones.

v. Apply inverse stationary wavelet transform to the threshold wavelet coefficients to obtain the de-noised EEG signal [11].

\section{ARTIFACT IDENTIFICATION}

There are several methods to identify automatically the artifact zones. When application requires real-time removal of artifacts or when calibration trials cannot be conducted owing to various constraints, method like ICA, regression becomes unsuitable. When accurate detection of these artifacts by singular observation of the time or frequency domains fails, wavelet transform maintaining the Integrity of the Specifications can be used to study the time-frequency maps of the EOG contaminated EEG. From the number of 'approximate' coefficients obtained at each level of wavelet decomposition, the approximate coefficients in the form aj $+1, a j$, aj- 1 is selected. Normally, every spike contains three coefficients. Next spike identification starts with aj+1, treated as aj-1and checks the next two coefficients. Based on this arrangement, the spikes in the contaminated EEG have to be identified. The decomposition level is restricted to six, in order to have a reasonable computational complexity. The mother wavelet should be chosen in such a way that it better approximates and captures the artifacts in the noisy EEG signal. Coiflet (Coif5) wavelet has been chosen as the basis function, since it resembles the shape of the eye movement [5]. 


\section{EOG AND EMG CORRECTION USING ADAPTIVE THRESHOLDING OF WAVELET COEFFICIENTS}

Wavelet Thresholding, Wavelet Shrinkage, and Non-linear Shrinkage are widely used terms for wavelet domain denoising. Principally, Donoho et al have developed denoising by thresholding in wavelet domain. In wavelet domain, larger coefficients correspond to the signal, and smaller ones represent mostly noise. The denoised data is obtained by inversetransforming the suitably thresholded, or shrunk coefficients. Algorithm for denoising is written as

- Compute $\mathrm{i}$ iterations of wavelet transformation on y to obtain the transformed vector $y$. Call the approximation portion as 1 and detail portion as $\mathrm{d}$.

- Apply the threshold rule to the detail portion $\mathrm{d}$ of $\mathrm{y}$. The rules will either "shrink" or set 0 values to detail.

- Rejoin the modified portion with the original portion of $y$ to form a modified transform vector $\hat{y}=[1 \mid d]$

- Compute i iterations of inverse wavelet transformation of $\hat{\mathrm{y}}$ to obtain $\mathrm{v}$. The $\mathrm{N}$ vector $\mathrm{v}$ should be denoised version of $\hat{g}$.

- $\quad \mathrm{E}(\|\mathrm{g}-\hat{\mathrm{g}}\|) 2$ is used to determine the effectiveness of the denoising method.

The standard thresholding of wavelet coefficients is governed mainly by either 'hard' or 'soft' thresholding function [6].

The hard thresholding function is given as:

$$
\begin{aligned}
& \mathrm{z}=\operatorname{hard}(\mathrm{w})=\mathrm{w},|\mathrm{w}|>\lambda, \text { and } \\
& \mathrm{z}=\operatorname{hard}(\mathrm{w})=0,|\mathrm{w}|<=\lambda
\end{aligned}
$$

where, $\mathrm{w}$ and $\mathrm{z}$ are the input and output wavelet coefficients respectively. $\lambda$ is a threshold value selected.

Similarly, soft thresholding function is given as:

$$
\begin{gathered}
\mathrm{z}=\operatorname{soft}(\mathrm{w})=\operatorname{sgn}(\mathrm{w}) \cdot \max (|\mathrm{w}|-\lambda, 0),|\mathrm{w}|>\lambda \text {, and } \\
\mathrm{z}=\operatorname{soft}(\mathrm{w})=0,|\mathrm{w}|<=\lambda
\end{gathered}
$$

In hard thresholding, the wavelet coefficients (at each level) below threshold $\lambda$ are made zero and coefficients above threshold are not changed whereas in soft thresholding, the wavelet coefficients are shrunk towards zero by an offset $\lambda$. Generally soft thresholding gives fewer artifacts and preserves the smoothness. The choice of threshold value is very crucial for a given signal for denoising. In this proposal, the thresholding algorithm employed is Stein's Unbiased Risk Estimate (SURE). Donoho et al. introduced various shrinkage rules based on different threshold values and thresholding functions such as 'VisuShrink' with fixed universal threshold $\lambda=\sigma \sqrt{2 \log 2 n}$ and 'another method based on Stein's Unbiased Risk Estimator (SURE), which is called SUREShrink. SureShrink suppresses noise by thresholding the wavelet coefficients. A threshold level is assigned to each level by the principle of minimizing the SURE threshold estimates. The computational effort of the procedure is in the order of $\mathrm{N} \cdot \log (\mathrm{N})$ as a function of sample size N. SureShrink is smoothness adaptive i.e if the unknown function contains jumps, the reconstruction also does and if the unknown function has a smooth piece, the reconstruction is as smooth as the mother wavelet will allow.
SureShrink has the following ingredients:

- Discrete Wavelet Transform of Noisy data. The N noisy data are transformed via the discrete wavelet transform, to obtain noisy wavelet coefficients (yj,k).

- Thresholding of Noisy wavelet Coefficients. Let $\eta \mathrm{t}(\mathrm{y})=\operatorname{sgn}(\mathrm{y})(|\mathrm{y}|-\mathrm{t})+$, which sets to zero data $\mathrm{y}$ below $\mathrm{t}$ in absolute value, and which pulls the other data towards the origin by an amount $t$. The wavelet coefficients $\mathrm{yj}, \mathrm{k}$ are subject to soft threshold with a level dependent threshold level $t * j$.

- Stein's Unbiased Estimate of Risk for Threshold Choice. The level-dependent thresholds are arrived at by regarding the different resolution levels (different $j$ ) of the wavelet transform as independent multivariate normal estimation problems. Within one level (fixed $\mathrm{j}$ ) one has data yj,k $=w j, \mathrm{k}+\varepsilon$ zj,k $, \mathrm{k}=0, \ldots ., 2 \mathrm{j}$-1and one wishes to estimate (wj,k) $2 \mathrm{j}-1 \mathrm{k}=0$.

- $\quad$ Stein's Unbiased Estimate of Risk for $\theta k(t)=\eta t(y j, k)$ gives an estimate of the risk for a particular threshold value $\mathrm{t}$; minimizing this give a selection of the threshold level $\mathrm{j}$.

Stein's theorem gives an alternative way to write the Mean Square Error (MSE), which is a method for estimating the error in an unbiased fashion.

In statistics, Stein's unbiased risk estimate (SURE) is an unbiased estimator of the mean-squared error of a given estimator. In other words, it provides an indication of the accuracy of a given estimator. This is important since, in deterministic estimation, the true mean-squared error of an estimator generally depends on the value of the unknown parameter, and thus cannot be determined completely. Then, Stein's unbiased risk estimate is given by

$$
\operatorname{SURE}(\mathrm{h})=\|\theta\|^{2}+\|\mathrm{h}(\mathrm{x})\|^{2}+2 \sigma^{2} \sum_{\mathrm{i}=1}^{\mathrm{n}} \frac{\partial \mathrm{h}_{\mathrm{i}}}{\partial \mathrm{x}_{\mathrm{i}}}-2 \sum_{\mathrm{i}=1}^{\mathrm{n}} \mathrm{x}_{\mathrm{i}} \mathrm{h}_{\mathrm{i}}(\mathrm{x})
$$

Where $\theta$ is a parameter vector from noisy measurements.

Here hi(x) is the ith component of the vector $\mathrm{h}$. The importance of SURE is that, it is an unbiased estimate of the mean-squared error of $h(x)$, i.e.

$$
\mathrm{E}\{\operatorname{SURE}(\mathrm{h})\}=\operatorname{MSE}(\mathrm{h})
$$

Thus, minimizing SURE can be expected to minimize the MSE. Except for the first term in SURE, which is identical for all estimators, there is no dependence on the unknown parameter $\theta$ in the expression for SURE above. Thus, it can be manipulated (e.g., to determine optimal estimation settings) without knowledge of $\theta$.

\section{RESULTS AND DISCUSSION}

In the proposed system three types of artifacts are simulated

The three different types of artifacts generated here are Eye Blink Artifact, Eye Ball movement Artifact and Muscle Artifact, which arise due to blinking, clenching or other facial expressions

(1) Eye blink time courses using random noise band-pass filtered (FIR) between 1 and $3 \mathrm{~Hz}$ were been modeled. For the eyeball movement artifacts, random noise 
band-pass filtered (FIR) between 3and $16 \mathrm{~Hz}$ were generated.

(2) Temporal muscle artifacts using random noise bandpass filtered (FIR) between 20 and $60 \mathrm{~Hz}$ were been modeled.

These types of artifacts were produced using Matlab. Applying stationary wavelet transforms to the contaminated EEG signals and decomposes it up to six levels with Coiflet (Coif5) as a basis function. The denoising technique employed at this stage is SURE by fixing a threshold value and thresholding function. The last stage is to obtain the de-noised EEG signal. Apply inverse stationary wavelet transform to the threshold wavelet coefficients to obtain the de-noised EEG signal.

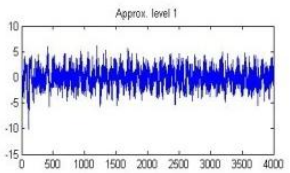

Appox: level 2

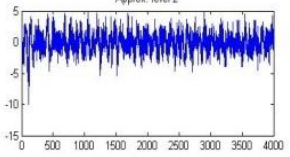

Appox leerel 3
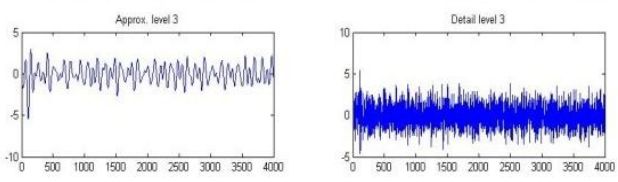

Fig (2) shows the 'approximation' and 'detail 'plot for the contaminated EEG signal
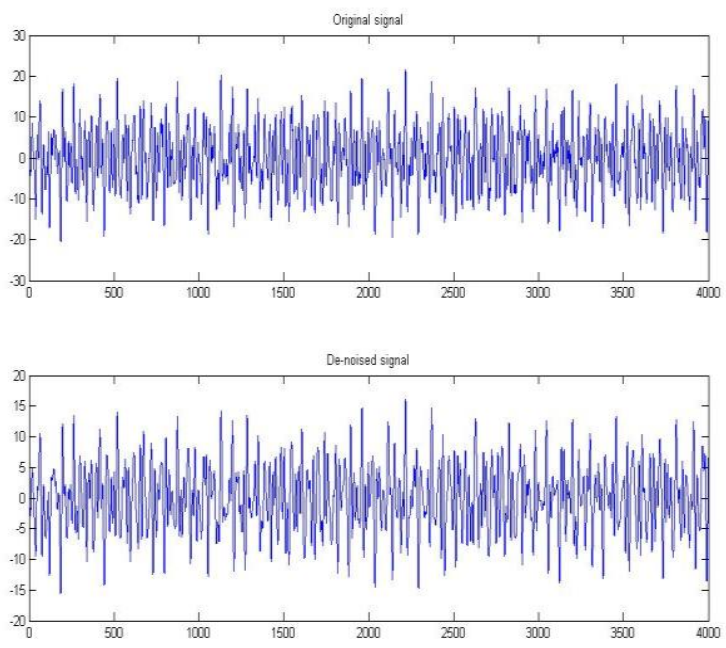

Fig (3) shows the de-noising of EEG signal is carried out by using different combinations of threshold limit, threshold function and window sizes.

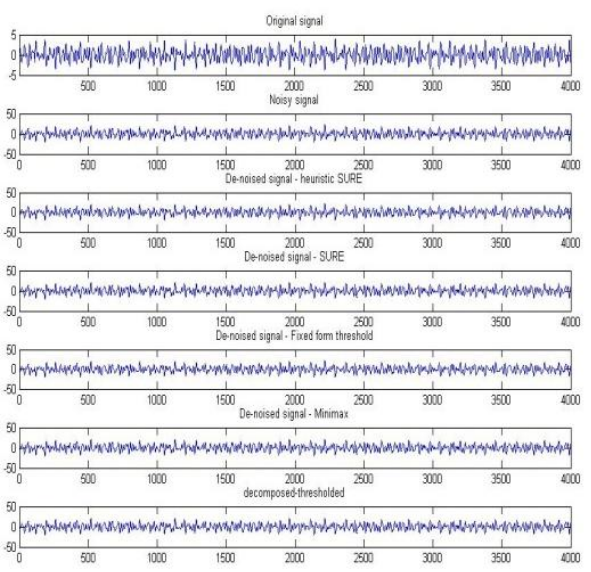

Fig (4) shows the Power Spectral Density of Contaminated signal

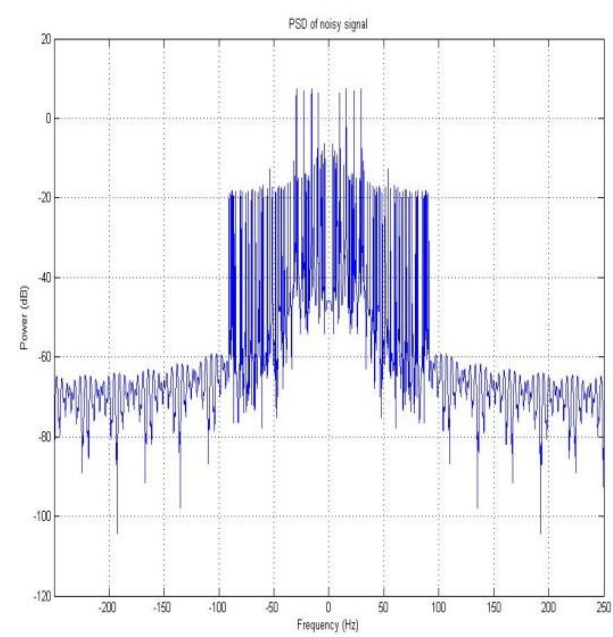

Fig (5) shows the Power Spectral density of artifact Free signal

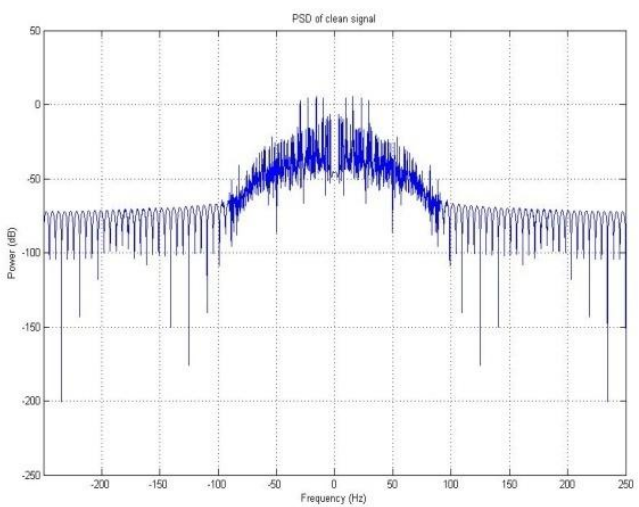

Fig (6) shows the Frequency correlation plot between noisy and De-noised data. 


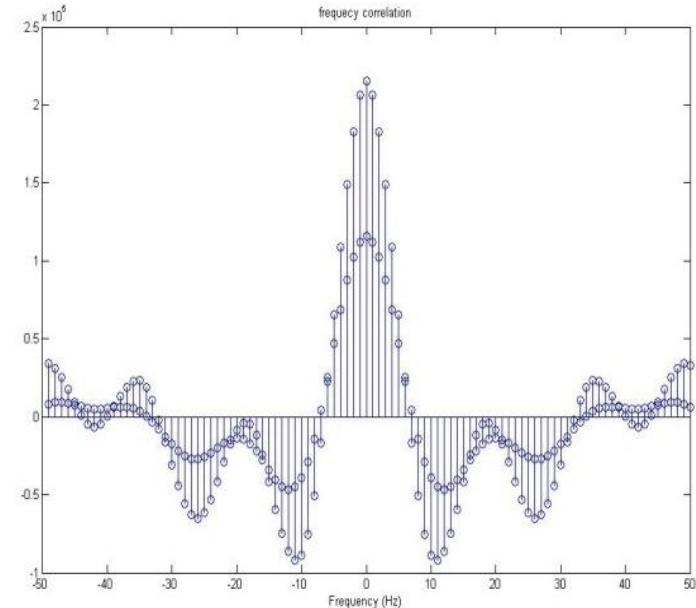

Fig (7) shows the Frequency correlation plot between noisy and De-noised data.

Artifacts in EEG signals are considerably reduced using the proposed method. The test bed used in our proposal was simulated data and for further augmentation, the trial could be carried on real time data obtained from hospitals.

\section{CONCLUSION}

A method to identify the ocular and muscular artifact spike zones through wavelet transform is proposed and SURE thresholding is applied to the artifact zones. Adaptive thresholding applied only to the artifact zone, does not attenuate the low frequency components and also preserves the shape of the EEG signal in the non-artifact zones, which is of very much importance in clinical diagnosis. The proposed method is to minimize the complexity of the work and easily identify the artifact zones for removing the artifacts. The metrics used to depict the performance in the proposed paper are Spectral Power Density and Correlation plots. Suppression of ocular artifacts and muscular artifacts proved to be good. It is concluded that the proposed method gives less complexity and easier to removal of the artifacts with the help of wavelet decomposition and is an efficient technique for improving the quality of EEG signals in biomedical analysis. Further, this method has been tested over simulated data, the same holds good to be applied for real time data.

\section{REFERENCES}

[1] R R Coifman and D L Donoho, 'Translation Invariant Denoising', in A Antoniadis (editor): Wavelets and Statistics, Springer Verlag, 1995.

[2] C Sidney Burrus, R A Gopinath, and Haitao Guo, 'Introduction to Wavelets and Wavelet Transforms: A Primer', Prentice Hall, NJ, 1998

[3] V. J. Samar, Ajit Bopardikar, Raghuveer Rao, and Kenneth Swartz, "Wavelet analysis of neuroelectric waveforms: A conceptual tutorial “, Brain and Language, 66, (1999), pp.7-60

[4] R.J. Croft and R.J. Barry, "Removal of ocular artifact from the EEG: a review ", Clinical Neurophysiology, Vol. 30, No.1, (2000), pp. 5 - 19.

[5] V. Krishnaveni, S. Jayaraman, S. Aravind, V. Hariharasudhan, K. Ramadoss, "Automatic identification and Removal of ocular artifacts from EEG using Wavelet transform ", Measurement Science Review, Vol. 6, No.4, (2006), pp.45-57.

[6] P. Senthilkumaret al," Removal of Ocular Artifacts in the EEG through Wavelet Transform without using an EOG Reference Channel", Int. J. Open Problems Compt. Math., Vol. 1, No. 3, December 2008, pp.188-200.

[7] M. Bedeeuzzaman, O. Farooq and Y. U. Khan, "EEG signal classification for seizure analysis using statistical measures”, Proc. EIP 2009, Vol.1, Pp. 366-372, 2009.

[8] T. Gandhi, B. K. Panigrahi, M. Bhatia and S. Anand, "Expert model for detecton of epileptic activity in EEG signature", Expert Systems with Applications, Vol. 37, No. 4, Pp. 3513-3520, 2010.

[9] E. D., Übeyli, "Combined neural network model employing wavelet coefficients for EEG signals clasification", Digital Signal Processing, Vol. 19, Pp. 297-308, 2009.

[10] A. M. Lima, A. L. V. Coelho, S. Chagas, "Automatic EEG signal classification for epilepsy diagnosis with Relevance Vector Machines," Expert Systems with Applications, Vol. 36, Pp. 10054-10059, 2009.

[11] Mukul, M.K. and Matsuno, F., "EEG de-noising based on wavelet-transforms and Extraction of Sub-band Components related to movement imagination", ICCASSICE, Pp. 1605-1610, 2009. 